International Journal of Pure and Applied Mathematics

Volume 90 No. 4 2014, 407-411

ISSN: 1311-8080 (printed version); ISSN: 1314-3395 (on-line version)

url: http://www.ijpam.eu

doi: http://dx.doi.org/10.12732/ijpam.v90i4.2

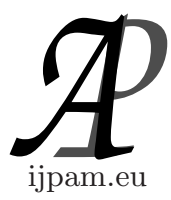

\title{
A NOTE ON THE HYPERCOMPLEX RIEMANN-CAUCHY LIKE RELATIONS FOR QUATERNIONS AND LAPLACE EQUATIONS
}

\author{
J.A.P.F. Marão ${ }^{1}$, M.F. Borges ${ }^{2} \S$ \\ ${ }^{1}$ Department of Mathematics \\ Federal University of Maranhão - São Luís-MA \\ 65085-580, Maranhão, BRAZIL \\ ${ }^{2}$ UNESP - São Paulo State University \\ S.J. Rio Preto Campus \\ 15054-000, São José do Rio Preto, BRAZIL
}

\begin{abstract}
In this Note it is worked out a new set of Laplace-Like equations for quaternions through Riemann-Cauchy hypercomplex relations otained earlier [1]. As in the theory of functions of a complex variable, it is expected that this new set of Laplace-Like equations might be applied to a large number of Physical problems, providing new insights in the Classical Fields Theory.
\end{abstract}

AMS Subject Classification: 30G99, 30E99

Key Words: quaternions, Laplace's equations, Riemann-Cauchy relations

\section{Cauchy-Riemann Equations (Functions of One Complex Variable)}

In order to fix ideas will be considered theorem that relates the partial derivatives for the case of a function $f(z)$ of a complex variable $f(z)=u(x, y)+i v(x, y)$ [2], which here will be called Riemann-Cauchy conditions. These relations say that the first order partial derivatives of functions $u(x, y)$ and $v(x, y)$ satisfy relations according to the following theorem:

Received: May 20, 2013

(C) 2014 Academic Publications, Ltd. url: www.acadpubl.eu

${ }^{\S}$ Correspondence author 
Theorem 1. Is $f(z)=u(x, y)+i v(x, y)$ a function defined and continues in a neighborhood of the point $z=x+y i$ and differentiable at $z$. Then the partial derivatives of the first order of $u(x, y)$ and $v(x, y)$ exist and satisfy the relations:

$$
\begin{gathered}
\frac{\partial u(x, y)}{\partial x}=\frac{\partial v(x, y)}{\partial y}, \\
\frac{\partial u(x, y)}{\partial y}=-\frac{\partial v(x, y)}{\partial x} .
\end{gathered}
$$

Thus, if $f(z)$ is analytic in a domain $\Gamma$, its partial derivatives exist and satisfy the set of relations (1) and (2) over all point in Gamma. Moreover, with the above functions class $C^{2}$ using Schwartz's Theorem for partial derivatives immediately follows the following equations:

$$
\begin{aligned}
& \frac{\partial^{2} u}{\partial x^{2}}+\frac{\partial^{2} u}{\partial y^{2}}=0 \\
& \frac{\partial^{2} v}{\partial x^{2}}+\frac{\partial^{2} v}{\partial y^{2}}=0
\end{aligned}
$$

the above equations are called Laplace's equations.

\section{Cauchy-Riemann Conditions for Quaternionic Functions}

Now we may consider a set of conditions presented as the Riemann-Cauchy like relations for quaternionic functions. It follows the Theorem [1]:

Theorem 2. For any pair pontis $a$ and $b$ and any path joining them simply conect subdomain of the four-dimmensional space, the integral $\int_{a}^{b} f d q$ is independent form the given path if and only if there is a function $F=$ $F_{1}+F_{2} i+F_{3} j+F_{4} k$ such that $\int_{\mathrm{a}}^{\mathrm{b}} f d q=F(a)-F(b)$, and satisfying the following relations:

$$
\begin{gathered}
\frac{\partial F_{1}}{\partial x_{1}}=\frac{\partial F_{2}}{\partial x_{2}}=\frac{\partial F_{3}}{\partial x_{3}}=\frac{\partial F_{4}}{\partial x_{4}}, \\
\frac{\partial F_{2}}{\partial x_{1}}=-\frac{\partial F_{1}}{\partial x_{2}}=-\frac{\partial F_{3}}{\partial x_{4}}=\frac{\partial F_{4}}{\partial x_{3}} \\
\frac{\partial F_{3}}{\partial x_{1}}=-\frac{\partial F_{1}}{\partial x_{3}}=-\frac{\partial F_{2}}{\partial x_{4}}=\frac{\partial F_{4}}{\partial x_{2}}, \\
\frac{\partial F_{4}}{\partial x_{1}}=\frac{\partial F_{1}}{\partial x_{4}}=-\frac{\partial F_{2}}{\partial x_{3}}=-\frac{\partial F_{3}}{\partial x_{2}} .
\end{gathered}
$$


Proof. The proof of this theorem can be analyzed in greater detail in [1].

\section{The Laplace's Equations}

In this section we show that a new set of hypercomplex Laplace equations may be generated in four dimensions, through the use of Riemann-Cauchy like relations [1] Therefore, the functions that make up the quaternionic function, depend on $x_{1}, x_{2}, x_{3}$ and $x_{4}$ and are supposed to be of class $C^{2}$ and thus the Schwartz's theorem is valid.

Theorem 3. Let $f(q)$ is an quaternionic function. If $f(q)$ is of class $C^{2}$ and satisfies the Cauchy-Riemann conditions, then

$$
\begin{aligned}
& \Delta f_{1}=0, \\
& \Delta f_{2}=0, \\
& \Delta f_{3}=0, \\
& \Delta f_{4}=0 .
\end{aligned}
$$

Demonstration: The first step to obtain the Laplace equation is the derivation of equations (5), (6), (7) and (8) over $x_{1}, x_{2}, x_{3}$ and $x_{4}$. That will be done as follows: Firstly, in deriving the conditions of equation (5), we have:

$$
\begin{aligned}
& \frac{\partial^{2} F_{1}}{\partial x_{1}^{2}}=\frac{\partial^{2} F_{2}}{\partial x_{1} \partial x_{2}}=\frac{\partial^{2} F_{3}}{\partial x_{1} \partial x_{3}}=\frac{\partial^{2} F_{4}}{\partial x_{1} \partial x_{4}} \\
& \frac{\partial^{2} F_{1}}{\partial x_{1} \partial x_{2}}=\frac{\partial^{2} F_{2}}{\partial x_{2}^{2}}=\frac{\partial^{2} F_{3}}{\partial x_{2} \partial x_{3}}=\frac{\partial^{2} F_{4}}{\partial x_{2} \partial x_{4}} \\
& \frac{\partial^{2} F_{1}}{\partial x_{3} \partial x_{1}}=\frac{\partial^{2} F_{2}}{\partial x_{3} \partial x_{2}}=\frac{\partial^{2} F_{3}}{\partial x_{3}^{2}}=\frac{\partial^{2} F_{4}}{\partial x_{4} \partial x_{3}} \\
& \frac{\partial^{2} F_{1}}{\partial x_{1} \partial x_{4}}=\frac{\partial^{2} F_{2}}{\partial x_{4} \partial x_{2}}=\frac{\partial^{2} F_{3}}{\partial x_{4} \partial x_{3}}=\frac{\partial^{2} F_{4}}{\partial x_{4}^{2}}
\end{aligned}
$$

In deriving the conditions of equation (6), we have:

$$
\begin{gathered}
\frac{\partial^{2} F_{2}}{\partial x_{1}^{2}}=-\frac{\partial^{2} F_{1}}{\partial x_{1} \partial x_{2}}=-\frac{\partial^{2} F_{3}}{\partial x_{1} \partial x_{4}}=\frac{\partial^{2} F_{4}}{\partial x_{1} \partial x_{3}}, \\
\frac{\partial^{2} F_{2}}{\partial x_{1} \partial x_{2}}=-\frac{\partial^{1} F_{1}}{\partial x_{2}^{2}}=-\frac{\partial^{2} F_{3}}{\partial x_{2} \partial x_{4}}=\frac{\partial^{2} F_{4}}{\partial x_{3} \partial x_{2}}, \\
\frac{\partial^{2} F_{2}}{\partial x_{3} \partial x_{1}}=-\frac{\partial^{2} F_{1}}{\partial x_{3} \partial x_{2}}=-\frac{\partial^{2} F_{3}}{\partial x_{3} \partial x_{4}}=\frac{\partial^{2} F_{4}}{\partial x_{3}^{2}}, \\
\frac{\partial^{2} F_{2}}{\partial x_{4} \partial x_{1}}=-\frac{\partial^{2} F_{1}}{\partial x_{4} \partial x_{2}}=-\frac{\partial^{2} F_{3}}{\partial x_{4}^{2}}=\frac{\partial^{2} F_{4}}{\partial x_{4} \partial x_{3}} .
\end{gathered}
$$


In deriving the conditions of equation (7), we obtain that:

$$
\begin{gathered}
\frac{\partial^{2} F_{3}}{\partial x_{1}^{2}}=-\frac{\partial^{2} F_{1}}{\partial x_{1} \partial x_{3}}=-\frac{\partial^{2} F_{2}}{\partial x_{1} \partial x_{4}}=\frac{\partial^{2} F_{4}}{\partial x_{1} \partial x_{2}}, \\
\frac{\partial^{2} F_{3}}{\partial x_{1} \partial x_{2}}=-\frac{\partial^{1} F_{1}}{\partial x_{2} \partial x_{3}}=-\frac{\partial^{2} F_{2}}{\partial x_{2} \partial x_{4}}=\frac{\partial^{2} F_{4}}{\partial x_{2}^{2}}, \\
\frac{\partial^{2} F_{3}}{\partial x_{3} \partial x_{1}}=-\frac{\partial^{2} F_{1}}{\partial x_{3}^{2}}=-\frac{\partial^{2} F_{2}}{\partial x_{4} \partial x_{3}}=\frac{\partial^{2} F_{4}}{\partial x_{3} \partial x_{2}}, \\
\frac{\partial^{2} F_{3}}{\partial x_{1} \partial x_{4}}=-\frac{\partial^{2} F_{1}}{\partial x_{4} \partial x_{3}}=-\frac{\partial^{2} F_{2}}{\partial x_{4}^{2}}=\frac{\partial^{2} F_{4}}{\partial x_{4} \partial x_{2}} .
\end{gathered}
$$

And finally in deriving the conditions of equation (8), we have:

$$
\begin{gathered}
\frac{\partial^{2} F_{4}}{\partial x_{1}^{2}}=\frac{\partial^{2} F_{1}}{\partial x_{1} \partial x_{4}}=-\frac{\partial^{2} F_{2}}{\partial x_{1} \partial x_{3}}=-\frac{\partial^{2} F_{3}}{\partial x_{1} \partial x_{2}}, \\
\frac{\partial^{2} F_{4}}{\partial x_{1} \partial x_{2}}=\frac{\partial^{2} F_{1}}{\partial x_{2} \partial x_{4}}=-\frac{\partial^{2} F_{2}}{\partial x_{2} \partial x_{3}}=-\frac{\partial^{2} F_{3}}{\partial x_{2}^{2}}, \\
\frac{\partial^{2} F_{4}}{\partial x_{3} \partial x_{1}}=\frac{\partial^{2} F_{1}}{\partial x_{3} \partial x_{4}}=-\frac{\partial^{2} F_{2}}{\partial x_{3}^{2}}=-\frac{\partial^{2} F_{3}}{\partial x_{3} \partial x_{2}}, \\
\frac{\partial^{2} F_{4}}{\partial x_{1} \partial x_{4}}=\frac{\partial^{2} F_{1}}{\partial x_{4}^{2}}=-\frac{\partial^{2} F_{2}}{\partial x_{4} \partial x_{3}}=-\frac{\partial^{2} F_{3}}{\partial x_{4} \partial x_{2}} .
\end{gathered}
$$

In correlating those groups of partial derivatives (9), (10), (11) and (12), immediately follow the Laplace Equations:

$$
\begin{aligned}
& \frac{\partial^{2} F_{1}}{\partial x_{1}^{2}}+\frac{\partial^{2} F_{1}}{\partial x_{2}^{2}}+\frac{\partial^{2} F_{1}}{\partial x_{3}^{2}}+\frac{\partial^{2} F_{1}}{\partial x_{4}^{2}}=0, \\
& \frac{\partial^{2} F_{2}}{\partial x_{1}^{2}}+\frac{\partial^{2} F_{2}}{\partial x_{2}^{2}}+\frac{\partial^{2} F_{2}}{\partial x_{3}^{2}}+\frac{\partial^{2} F_{2}}{\partial x_{4}^{2}}=0, \\
& \frac{\partial^{2} F_{3}}{\partial x_{1}^{2}}+\frac{\partial^{2} F_{3}}{\partial x_{2}^{2}}+\frac{\partial^{2} F_{3}}{\partial x_{3}^{2}}+\frac{\partial^{2} F_{3}}{\partial x_{4}^{2}}=0,
\end{aligned}
$$

and

$$
\frac{\partial^{2} F_{4}}{\partial x_{1}^{2}}+\frac{\partial^{2} F_{4}}{\partial x_{2}^{2}}+\frac{\partial^{2} F_{4}}{\partial x_{3}^{2}}+\frac{\partial^{2} F_{4}}{\partial x_{4}^{2}}=0
$$

Therefore, it is more simplified manner, the set of equations appears below:

$$
\begin{aligned}
& \Delta f_{1}=0 \\
& \Delta f_{2}=0, \\
& \Delta f_{3}=0 \\
& \Delta f_{4}=0
\end{aligned}
$$


where,

$$
\begin{aligned}
& f_{1}=f_{1}\left(x_{1}, x_{2}, x_{3}, x_{4}\right), \\
& f_{2}=f_{2}\left(x_{1}, x_{2}, x_{3}, x_{4}\right), \\
& f_{3}=f_{3}\left(x_{1}, x_{2}, x_{3}, x_{4}\right), \\
& f_{4}=f_{4}\left(x_{1}, x_{2}, x_{3}, x_{4}\right) .
\end{aligned}
$$

\section{Conclusion}

In this note it is showed the feasibility of obtaining the equations of Laplace through the Cauchy-Riemann conditions for quaternions. This fact will allow the relationship between equations that can explain many physical phenomena. You can also use the above equations as a way of stating a theorem for harmonic functions for quaternions that satisfy the conditions of Cauchy. It is worth mentioning the importance of [1] in performing all relations used in this work.

\section{References}

[1] J.M. Machado, M.F. Borges, New ramrks on the differenciability of hypercomplex functions, International Journal of Pure and Applied Mathematics, 8, No. 1 (2002), 85-101.

[2] Kunihiko Kodaira, Complex Analysis, Cambridge Studies in Advanced Mathematics, Cambridge University Press Cambridge (2007), 406pp. 
